\title{
How the Performance of a Superconducting Magnet is affected by the Connection between a Small Cooler and the Magnet *
}

\author{
Michael A. Green \\ Lawrence Berkeley National Laboratory \\ Berkeley CA 94703, USA
}

September 2005

Presented at the 2005 Magnet Technology Conference (MT-19)

Genoa, Italy, 18 through 23 September 2005

Published in IEEE Transactions on Applied Superconductivity 16, No. 1, (2006)

\footnotetext{
* This work was performed at the Lawrence Berkeley National Laboratory with the support of the Office of High Energy Physics, United States Department of Energy under DOE contract DE-AC02-05CH11231. DOE funding for the US Neutrino Factory and Muon Collider Collaboration is gratefully acknowledged.
} 


\section{DISCLAIMER}

This document was prepared as an account of work sponsored by the United States Government.

While this document is believed to contain correct information, neither the United States Government nor any agency thereof, nor The Regents of the University of California, nor any of their employees, makes any warranty, express or implied, or assumes any legal responsibility for the accuracy, completeness, or usefulness of any information, apparatus, product, or process disclosed, or represents that its use would not infringe privately owned rights. Reference herein to any specific commercial product, process, or service by its trade name, trademark, manufacturer, or otherwise, does not necessarily constitute or imply its endorsement, recommendation, or favoring by the United States Government or any agency thereof, or The Regents of the University of California. The views and opinions of authors expressed herein do not necessarily state or reflect those of the United States Government or any agency thereof, or The Regents of the University of California. 


\title{
How the Performance of a Superconducting Magnet is affected by the Connection between a Small Cooler and the Magnet*
}

\author{
M. A. Green,
}

\begin{abstract}
As low temperature cryocoolers become more frequently used to cool superconducting magnets, it becomes increasingly apparent that the connection between the cooler and the magnet has an effect on the design and performance of the magnet. In general, the use of small coolers can be considered in two different temperature ranges; 1) from 3.8 to 4.8 K for magnet fabricated with LTS conductor and 2) from 18 to $35 \mathrm{~K}$ for magnets fabricated using HTS conductor. In general, both temperature ranges call for the use of a twostage cooler. The best method for connecting a cooler to the magnet depends on a number of factors. The factors include: 1) whether the cooler must be used to cool down the magnet from room temperature, 2) whether the magnet must have one or more reservoirs of liquid cryogen to keep the magnet cold during a loss of cooling, and 3 ) constraints on the distance from the cooler cold heads and the magnet and its shield. Two methods for connecting low temperature coolers to superconducting magnets have been studied. The first method uses a cold strap to connect the cold heads directly to the loads. This method is commonly used for cryogen-free magnets. The second method uses a thermal siphon and liquid cryogens to make the connection between the load being cooled and the cold head. The two methods of transferring heat from the magnet to the cooler low temperature cold head are compared for the two temperature ranges given above.
\end{abstract}

Index Terms-S/C Magnet, Small Coolers, Cooler Connection

\section{INTRODUCTION}

$\mathrm{T}$ he development of reliable small coolers that can cool magnets in the temperature range from 4.0 to $4.5 \mathrm{~K}$ has had profound effect on the ability to commercialize low temperature superconductors (LTS). High temperature superconductors (HTS) are now beginning to become commercially viable at temperatures from 20 to $35 \mathrm{~K}$. It is also attractive to cool HTS magnets using small coolers. In both cases, the coolers used to cool the magnets will be twostage coolers. Only at temperatures above 35 to $40 \mathrm{~K}$ does a single-stage cooler become attractive

The development of the so-called cryogen-free magnet technology has had a positive psychological effect on the magnet marketplace. It is likely (given the operating temperature range) that HTS magnets will be cryogen-free magnets. The case for cryogen-free magnets is not so evident when one looks at large LTS magnets, particularly those that use niobium-titanium as a conductor.

Manuscript received 19 September 2005. M. A. Green is from the Lawrence Berkeley Laboratory, Berkeley CA 94729, USA

*This work was also performed at the Lawrence Berkeley National Laboratory with the support of the Office of High Energy Physics, United States Department of Energy under DOE contract DE-AC02-05CH11231.
In general, intrinsically stable superconductors don't care how they are cooled. For magnets that have conductors that are not intrinsically stable the cooling method can matter as can the resistivity of the matrix material (usually copper) that contains the superconducting filaments.

The performance of a magnet is determined by whether the conductor is superconducting at the highest field point in the magnet. This point often corresponds to the hottest point in the magnet because it is often a point that is farthest from the cooling source. For all superconductors, the critical current $I_{c}$ is a function of the magnetic induction in the wire, the temperature, and the strain state in the conductor. With LTS such as alloy conductors like $\mathrm{Nb}-\mathrm{Ti}$ or A-15 conductors like $\mathrm{Nb}_{3}$ Sn the critical current $\mathrm{I}_{\mathrm{c}}$ can be calculated over a broad range of magnetic induction $B$, temperature $T$, and strain $\square$ provided one knows the critical current at a particular temperature, magnetic induction, and strain [1], [2].

With LTS conductors, the magnet conductor critical current $I_{c}$ is often plotted on a linear plot as a function of $B$ for various values of $T$. If one plots the magnetic induction at the high field point in the magnet as a function of the coil current, one gets the so-called load line curve for a magnet. Figure 1 shows the load line for the MICE focusing magnet in the flip and non-flip modes along with the conductor $I_{c}$ at $3.4 \mathrm{~K}, 4.2 \mathrm{~K}$, and $5.0 \mathrm{~K}$ [3]. The plot shows two operating points. The lower current points are the guaranteed design operating points for the magnets. The higher current points are at currents 20 percent higher than the lower current points. Both are the highest possible operating points for the MICE focusing magnet operating in the flip mode (with the two coils in series at opposite polarity) and non-flip mode (with the two coils in series at the same polarity) [3].

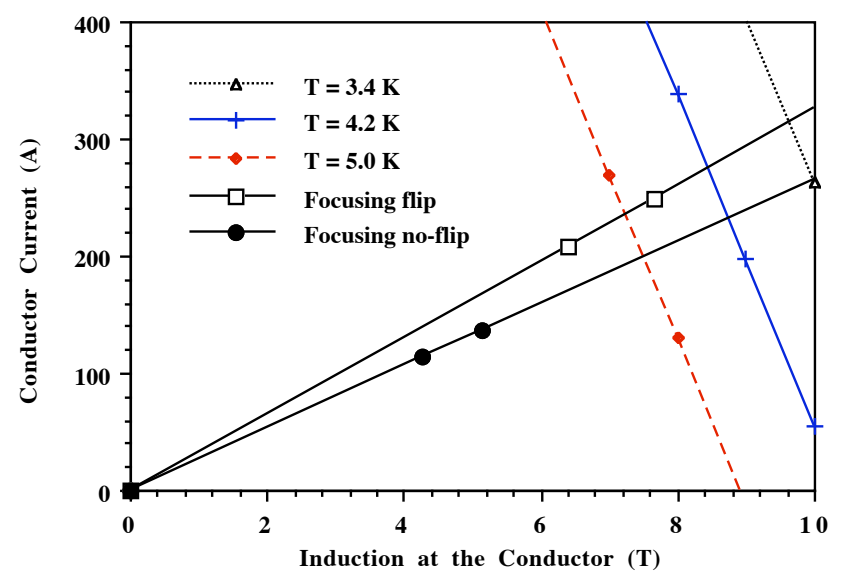

Figure 1. The load lines for the MICE focusing magnet operating in the flip and non-flip modes along with the $I_{c}$ as a function of $B$ for various $T$. 
Fig.1 illustrates the concept of the operating temperature margin for a superconducting magnet. In fig. 1, one can see that the highest current in the flip mode has a temperature margin of about $0.6 \mathrm{~K}$ when the magnet is at $4.2 \mathrm{~K}$. That means that the critical temperature of the conductor at the high field point is only about $4.8 \mathrm{~K}$. When on looks at the lower current point along the flip load line, one sees that the temperature margin is much higher (about $1.1 \mathrm{~K}$ ). The energy needed to quench the magnet is a function of the temperature margin. The higher the temperature margin the harder it is to quench the magnet.

There are three ways of increasing the temperature margin in a magnet that is cooled with a small cooler; 1) decrease the heat load $\mathrm{Q}_{\mathrm{L}}$ into the magnet and the cooler thus reducing the second stage cold head temperature, 2) reduce the temperature drop $\square \mathrm{T}_{1}$ between the hot spot in the magnet and the point where the cooling is applied to the magnet, and 3) reduce the temperature drop $\square T_{2}$ between the second stage cold head and the point where the cooling is applied to the magnet. It should be pointed out that decreasing the heat load into the magnet will reduce $\square T_{1}$ and $\square T_{2}$, but it is also important to tackle the reduction of $\square T_{1}$ and $\square T_{2}$ separately because there is a limit to the extent that one can reduce the overall heat load into the magnet.

\section{COOLER Performance AS A Function of LOAD}

Figure 2 shows the performance curves for a typical 1-W pulse tube cooler over a range of first-stage temperatures from 20 to $80 \mathrm{~K}$ and a range of second-stage temperatures from 0 to $25 \mathrm{~K}$ [4]. The minimum no load $(\mathrm{Q} 2=0)$ temperature for the cooler shown in Fig. 2 is from 2.5 to $3 \mathrm{~K}$ depending on the heat load Q1 into the first stage. The second-stage cold head temperature at a heat load of $20 \mathrm{~W}$ varies from $17 \mathrm{~K}$ to $23 \mathrm{~K}$ depending on the heat load Q1 applied to the first stage. A similar set of performance curves will apply for a typical 4 K Gifford McMahon (GM) cooler.

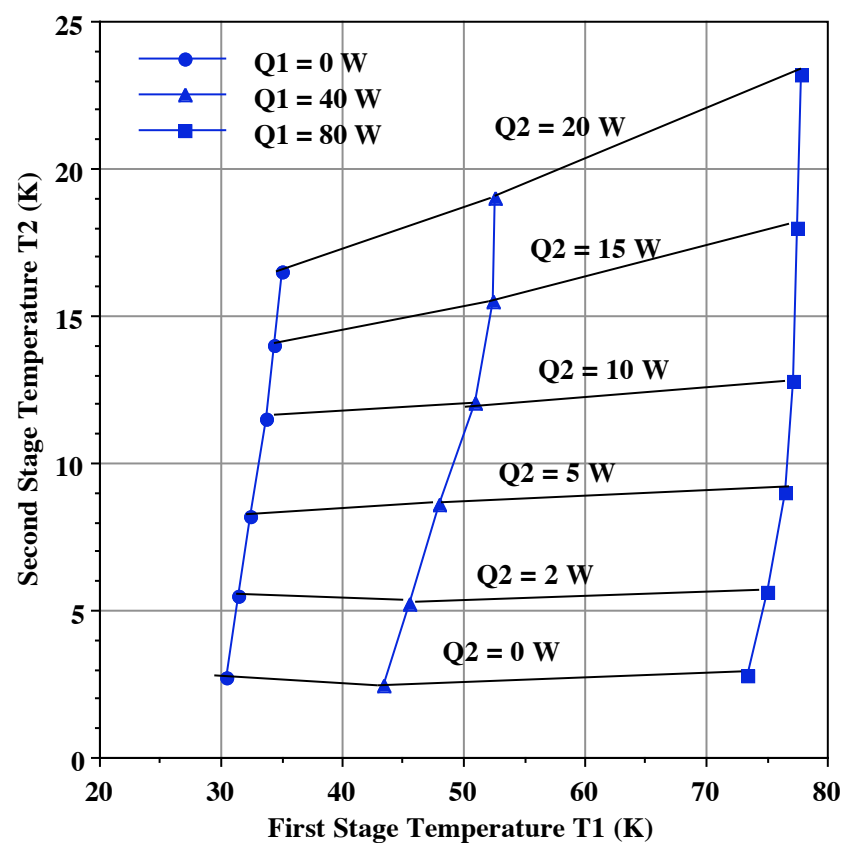

Figure 2 The temperature of the first-stage T1 and the second-stage T2 of a typical $1 \mathrm{~W}$ pulse tube cooler as a function of the first-stage heat load Q1 and the second-stage heat load Q2.
From Fig. 2, it is clear that reducing the heat load into the cooler second-stage cold head will reduce the temperature of that cold head. The first-stage cold head temperature is also strongly dependent on the heat load into the first-stage. Magnets that have continuously powered current leads will have a large heat load from the leads into the cooler firststage. The first-stage heat load, for a typical magnet, is dominated by heat from room temperature down the leads. In a well-designed magnet, a third to half of the heat into the second stage comes down the HTS leads from the first-stage of the cooler. The other heat loads into the second-stage of the cooler are also a strong function of the first-stage temperature. One way to reduce the heat load into both stages of the cooler is to reduce the current in the magnet.

\section{REDUCING THE TEMPERATURE DROP IN THE MAGNET}

One of the ways of reducing the temperature drop $\mathrm{CD}_{1}$ from the magnet hot spot (usually the high field point as well) is to reduce the overall heat load into the magnet. For LTS magnets this means reducing the first stage temperature, improving the insulation, reducing the conductivity of the cold mass supports, and reducing the conductivity of the HTS leads between the cooler first-stage and the magnet coil. In an HTS magnet, one also must also reduce the resistive heating within the magnet conductor. The resistive heating is a function of the quality of the superconductor as well as the operating temperature of the magnet.

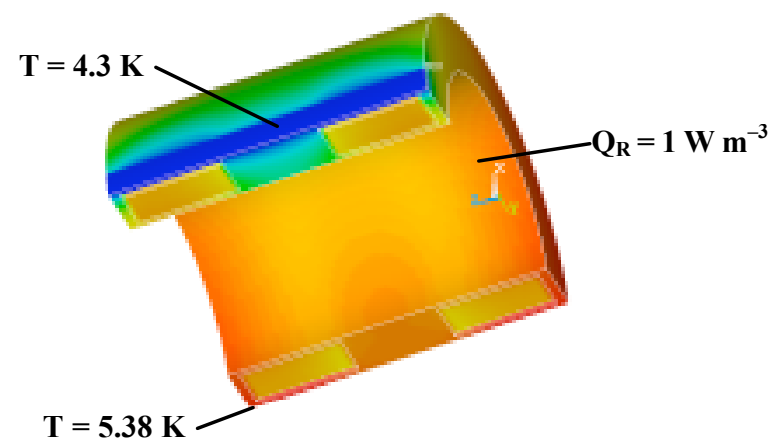

Figure 3. The temperature distribution within the MICE focusing magnet when a $100 \mathrm{~mm}$ wide strip on the outside of the magnet is at $4.3 \mathrm{~K}$.

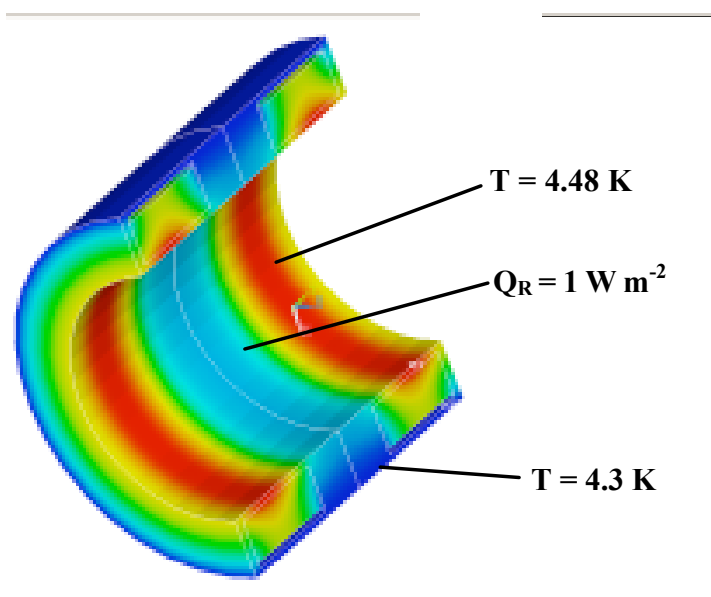

Figure 4. The temperature distribution within the MICE focusing magnet when the entire outside surface of the magnet is at $4.3 \mathrm{~K}$. 
Figure 3 shows the temperature distribution in the MICE focusing magnet cooled to $4.3 \mathrm{~K}$ along a $100 \mathrm{~mm}$ wide long line at the top of the magnet (where a cooler might be attached) [5]. Figure 4 shows the temperature distribution in the MICE focusing magnet when the entire outer surface is cooled to $4.3 \mathrm{~K}$ (the magnet is surrounded by liquid $\mathrm{He}$ ) [5]. In both cases the heat load applied to all of the other surfaces is $1.0 \mathrm{~W} \mathrm{~m}^{-3}$, the magnet case is made from 6061-T6aluminum, which has a thermal conductivity at $4.3 \mathrm{~K}$ of about $7.4 \mathrm{~W} \mathrm{~m}^{-1} \mathrm{~K}^{-1}$.

The temperature difference in Figure 3 is $1.08 \mathrm{~K}$ for a total heat flow into the magnet is $2.42 \mathrm{~W}$. The temperature difference in Figure 4 is $0.18 \mathrm{~K}$, but the total heat flow is reduced to $1.43 \mathrm{~W}$. One can reduce the temperature difference in both cases by changing the case material to a more conductive material or by reducing the heat input per unit area. In the example used, the case must be made from 6061T6-aluminum in order to carry large magnetic forces. Even if the heat load in both cases were reduced to $1 \mathrm{~W}$, the temperature difference for the case in Fig. 3 would be $0.45 \mathrm{~K}$, whereas the temperature difference for the case in Fig. 4 would be only $0.13 \mathrm{~K}$. It is clear that it is desirable to cool the entire outside surface of the magnet as shown in Fig. 4.

Increasing the 6061-T6-aluminum case temperature to $25 \mathrm{~K}$ (as with HTS magnets) will increase its thermal conductivity to about $35 \mathrm{~W} \mathrm{~m}^{-1} \mathrm{~K}^{-1}$. The thermal conductivity of the coil will also go up a factor of four. For a given heat load into the coil, the increase in thermal conductivity of the case and the coil means that the temperature rise in the magnet $\square \mathrm{T}_{1}$ is less of a problem at higher temperatures. HTS magnet are often more tolerant of large temperature changes.

\section{REDUCING THE TEMPERATURE DROP FROM THE MAGNET TO THE COOlER SECONd Stage COLd HeAd}

The temperature drop between the place on the magnet where cooling is applied and the cooler second-stage cold head $\square \mathrm{T}_{2}$ depends on the method used to transfer the heat to the cooler. Ideally, one wants to attach the cooler directly to the load being cooled. There are often sound engineering reasons why this can't be done. The reasons might include; 1) the cooler orientation is not optimum for maximum cooling; 2) the cooler interferes with the location of magnet leads, shields, or cold mass supports; 3 ) cooler vibration is an issue; and 4) cooler maintenance requires that the cooler be located physically away from the magnet.

A common way of connecting the cooler to the magnet is using a flexible copper strap (see Figure 5) [6]. The temperature drop along the strap $\square T$, is a function of the heat load $\mathrm{Q}$, the thermal conductivity of the strap $k\left(T_{A V E}\right)$ at the average temperature of the strap $T_{A V E}=\left(T_{1}+T_{2}\right) / 2$, the length of the strap $\ell$, and the cross-sectional area of the strap $A_{C S}$. An expression for the $\square \mathrm{T}$ along the strap is given as follows;

$$
\square T=T_{2} \square T_{1}=\frac{Q \ell}{k\left(T_{A V E}\right) A_{C S}}
$$

where

$$
k\left(T_{A V E}\right)=\frac{L\left(T_{1}+T_{2}\right)}{2 \square}
$$

where $\mathrm{L}$ is the Lorenz number $\left(\mathrm{L}=2.45 \times 10^{-8} \square \mathrm{WK}^{-2}\right)$, $\square$ is the strap electrical resistivity at $\mathrm{T}_{\mathrm{AVE}}, \mathrm{T}_{2}$ is the magnet temperature where the strap is attached, and $T_{1}$ is close to the $2^{\text {nd }}$-stage cold head temperature, which is a function of $Q$.

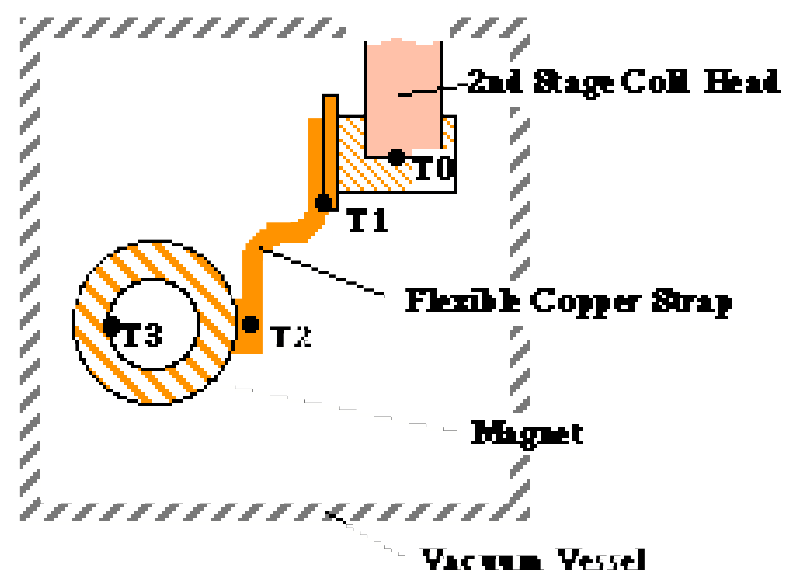

Figure 5. An illustration of the connection of the magnet to the cooler second-stage using a flexible copper strap.

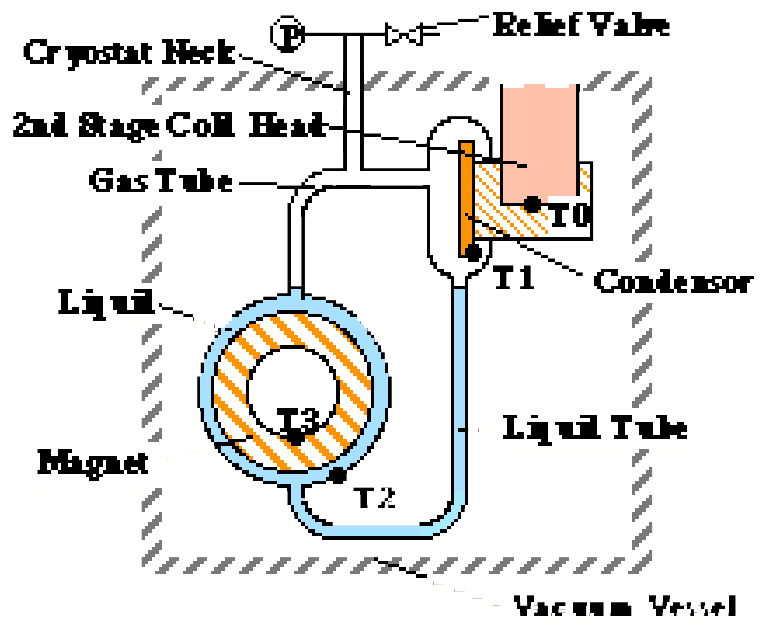

Figure 6. An illustration of the connection of the magnet to the cooler second-stage cold head using a thermal siphon.

Figure 6 illustrates the second method of connecting the cold head to the magnet using a thermal siphon. The thermal siphon consists of a boiling surface (the surface of the magnet or tubes connected to the magnet) and a condenser that is connected to the cooler second-stage cold head. The temperature drop between the condenser and the cold head $\left(T_{1}-T_{0}\right)$ must be made as small as possible. The temperature drop between the condenser and the magnet surface $\left(T_{2}-T_{1}\right)$ consists of two parts, a boiling part $\square \mathrm{T}_{\mathrm{B}}$ and a condensing part $\square \mathrm{T}_{\mathrm{C}}$. One can calculate the temperature drop between the magnet and the condenser using the following expression [7];

$$
T_{2} \square T_{1}=\square T_{B}+\square T_{C}
$$

where $\square \mathrm{T}_{\mathrm{C}}$ is calculated with the following expression [8];

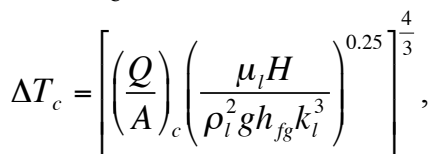

where $(\mathrm{Q} / \mathrm{A})_{\mathrm{c}}$ is the heat transferred per unit area across the condenser; $\square_{1}$ is the viscosity of the liquid; $H$ is the height of the condensing plate; $\square_{\mathrm{t}}$ is the density of the liquid; $\mathrm{g}$ is the acceleration of gravity; $h_{f g}$ is the heat of vaporization of the fluid; and $\mathrm{k}_{1}$ is the liquid thermal conductivity. For condensing liquid helium, $(\mathrm{Q} / \mathrm{A})_{\mathrm{c}}<40 \mathrm{~W} \mathrm{~m}^{-2}$ on the surface. Extending the condenser surface is nearly always a good idea. 
An expression for the temperature drop $\square \mathrm{T}_{\mathrm{b}}$ from a device to liquid helium through nucleate boiling at $4.2 \mathrm{~K}$ can be given by the following expression [7], [9];

$$
\square T_{B}=\frac{\square(Q / A)_{B}}{\square} \square T_{N B}
$$

where $(Q / A)_{B}$ is the heat transfer per unit area on the boiling surface (the magnet surface), $(Q / A)_{N B}$ is the peak nucleate boiling flux for the fluid from a surface facing upward (For helium at $4.2 \mathrm{~K}(Q / A)_{N B}=8000 \mathrm{~W} \mathrm{~m}^{-2}$.); and $\square T_{N B}$ is the peak temperature drop associated with peak nucleate boiling on a open surface facing upward (For helium $\square T_{N B}=0.8 \mathrm{~K}$.). In order to have a low value of $\square T_{B},(Q / A)_{B}<2 \mathrm{~W} \mathrm{~m}^{-2}$. If one designs the condenser so that $(Q / A)_{C}=40 \mathrm{~W} \mathrm{~m}^{-2}$ and $(Q / A)_{B}=2 \mathrm{~W} \mathrm{~m}^{-2}$ for the magnet the total temperature drop from the magnet to the cold head can be less than $0.05 \mathrm{~K}$.

It is useful to note that equations $2 \mathrm{a}$ and $2 \mathrm{~b}$ do not have any dependence on the distance between the magnet and the condenser surface attached to the cooler cold head. The pipes connecting the magnet cryostat to the condensation chamber must be large enough to carry the gas and liquid flow with a reasonably small pressure drop.

The thermal siphon method of reducing the temperature drop is particularly useful at $4 \mathrm{~K}$. This method can be used in the 20 to $30 \mathrm{~K}$ range if hydrogen or neon are used as fluids. At the higher temperature range, the temperature drop is usually less critical, so using a strap is often favored.

\section{Magnet CoOL Down}

There are two ways of cooling down the magnet. One can cool the magnet down through the copper strap or one can cool the magnet separately using liquid cryogens in tubes attached to the magnet.

Cryogen-free magnets are cooled down using the cooler alone. The conductance of the strap can adversely affect the cool down rate [6]. Since the cooler refrigeration is nearly linear with temperature, the temperature drop along the strap will reduce the cooler temperature. The conductance of the strap is like the conductance of a vacuum system in that strap conductance reduces the cooling rate as the conductance of a vacuum tube affects the effective pumping speed. A cryogenfree magnet should have a strap with maximum conductance for a more rapid cool down.

Cooling a magnet down using liquid cryogens can be accomplished in a short time (about 4 hours even for a magnet with a mass of several tons). The key to liquid cooling is getting rid of the liquid nitrogen before finishing cool down with liquid helium. The thermal siphon circuit can be part of the cool down circuit so that the space around the magnet is filled with liquid helium at the end of the cool down. Extra time has to be allotted for the insulation to come to equilibrium. This process can take up to 48 hours.

\section{Concluding Comments}

The connection of the magnet to the cooler is critical when the operating temperature margin for the magnet is small. The temperature margin is nearly always small with $\mathrm{Nb}-\mathrm{Ti}$ magnets, but it can be small for magnets fabricated from other LTS conductors. In general, the temperature margin is higher for an HTS magnet. Keeping the magnet at the lowest possible temperature is less important for HTS magnets.

The refrigeration delivered by a small cooler is often linear with temperature from $4 \mathrm{~K}$ on up. A cooler that delivers 1.0 to $1.5 \mathrm{~W}$ at $4.2 \mathrm{~K}$ will deliver as much as $20 \mathrm{~W}$ at $20 \mathrm{~K}$. Two-stage coolers are favored at temperatures below $30 \mathrm{~K}$. When on operates magnets at $30 \mathrm{~K}$ or below, the first stage of the cooler plays the key role of intercepting heat coming down the leads from room temperature. HTS leads must be used between the first and second stages of the cooler. Sometimes liquid reservoirs are used to provide back up cooling in the event of a power or cooler failure.

The temperature drops within the magnet are as important as the temperature drop from the magnet to the cooler. Cooling a $4 \mathrm{~K}$ magnet on the outside is a good way of reducing the temperature drop within the magnet.

The connection of the magnet to the cooler second-stage cold head is a key to reducing the temperature drop between the magnet hot spot (usually the high field point in the magnet) and the cold head. Connecting the cooler to the magnet through a copper strap makes sense when the magnet temperature margin is higher. At higher temperatures the strap thermal conductivity is higher so the temperature drop is lower for a given heat load. A thermal siphon is a good way of reducing the temperature drop between the magnet and the cold head, particularly at $4 \mathrm{~K}$. The thermal siphon will reduce the $\square \mathrm{T}$ between the magnet surface and the cold head to as low as $0.05 \mathrm{~K}$ at $4.2 \mathrm{~K}$. In all cases it is important to reduce the heat leak into the magnet as much as possible.

Cryogen-free magnets must be cooled down with a cooler. The cool down rate is limited by the conductance of the strap between the cooler and the magnet. Larger magnets and magnet with cryogens in them can be cooled down with liquid cryogens in a short time.

\section{REFERENCES}

[1] M. A. Green, "Calculating the Jc, B, T Surface for Niobium Titanium Using the Reduced State Model", IEEE Transactions on Magnetics 25, No. 2, p 2119, (1989)

[2] M. A. Green, "Calculating the $\mathrm{J}_{\mathrm{C}}, \mathrm{B}, \mathrm{T}$ Surface for Commercial Niobium Tin Conductors Using a Reduced State Model," Advances in Cryogenic Engineering 40, p 733 Plenum Publishing, New York, (1994)

[3] S. Q. Yang, M. A. Green, G. Barr, et al, "The Mechanical and Thermal Design for the MICE Focusing Solenoid Magnet System," IEEE Transactions on Applied Superconductivity 15, No. 2, p 1259, (2005),

[4] Private communication with Peter Gifford of Cryomech corporation concerning the the high temperature performance of their PT-410 and PT-810 pulsed tube coolers, (2005).

[5] M. A. Green and S. Q. Yang, "Heat Transfer into and within the 4.4 K Region and the $40 \mathrm{~K}$ Shields of the MICE Focusing and Coupling Magnets," Oxford University Physics Department Note 2, MICE Note 101, http://hep04.phys.itt.edu/cooldemo, (April 2004)

[6] M. A. Green, "Cooling the MICE Magnets using Small Cryogenic Coolers," Oxford University Physics Department Note 10, MICE Note 109, http://hep04.phys.itt.edu/cooldemo, (September 2004)

[7] M. A. Green, "The Integration of Liquid Cryogen Cooling and Cryocoolers with Superconducting Electronics Systems," Superconducting Science and Technology, Vol. 16, No. 12, p 1349, (Dec. 2003),

[8] Frank Kreith, Principles of Heat Transfer, International Textbook Co., Scranton PA. (1958)

[9] M. A. Green, D. R. Dietderich, S. Marks, et al, "Design Issues for Cryogenic Cooling of Short Period Superconducting Undulators," Advances in Cryogenic Engineering 49, p 783, (2004), 